\title{
A Shortest Path Searching Method with Area Limitation Heuristics
}

\author{
Feng $\mathrm{Lu}^{1}$ and Poh-Chin Lai ${ }^{2}$ \\ ${ }^{1}$ State Key Laboratory of Resources and Environmental Information System, \\ The Institute of Geographical Sciences and Natural Resources Research, \\ Chinese Academy of Sciences, Beijing 100101, P.R. China \\ lufalreis.ac.cn \\ ${ }^{2}$ Department of Geography, The University of Hong Kong, \\ Hong Kong SAR, P.R. China
}

\begin{abstract}
While heuristics based on geometric constructs of the networks would appear to improve performance of Dijkstra's algorithm, the fallacy of depreciated accuracy has been an obstacle to the wider application of heuristics in the search for shortest paths. The authors presented a shortest path algorithm that employs limited area heuristics guided by spatial arrangement of networks. The algorithm was shown to outperform other theoretically optimal solutions to the shortest path problem and with only little accuracy lost. More importantly, the confidence and accuracy levels were both controllable and predictable.
\end{abstract}

\section{Introduction}

Shortest path algorithms traditionally concerned topological or phase characteristics of a network and neglect the spatial or proximity characteristics. Such a viewpoint has resulted in a looping and radial approach to path searching which cannot prevent redundant searching even in situations when the destination nodes have been located. Some researchers have argued that the locational information (i.e. relative positions) of nodes can be employed in heuristics to inform the searching process [1][2]. Heuristics have been widely used in optimum path algorithms [3][4][5].

Most heuristics utilized local controlling strategies to guide the node search. They were efficient in locating approximately optimal one-to-one shortest paths but could not handle one-to-some or some-to-one shortest path problems.

The optimal path algorithms and heuristic strategies can be integrated to make a trade-off between efficiency and theoretical exactness, which is especially important for large-scale web applications or real time vehicular path finding. This paper highlights advantages of the integration using real-world networks of different complexity and connectedness.

\section{Real-World Networks in the Study}

We downloaded ten real-world networks representing road structures of varied complexity from http://www.fhwa.dot.gov/ and http://www.bts.gov/. The network set 
Table 1. Characteristics of real-world networks in the study

\begin{tabular}{cccccc}
\hline Network set & Alabama & Georgia & Pennsylvania & New York & Texas \\
Number of nodes & 952 & 2308 & 2640 & 3579 & 3812 \\
Number of arcs & 1482 & 3692 & 4183 & 5693 & 6340 \\
\hline Network set & California & Northeast & USA & Utah & Alabama \\
Number of nodes & 5636 & 14009 & Total & Detailed & Detailed \\
Number of arcs & 9361 & 19788 & 44077 & 72558 & 154517 \\
\hline
\end{tabular}

data were edited to topological correctness and then transformed into some custom-built ASCII files to store geometrical and topological information.

\section{Search Heuristics Based upon Geographic Proximity}

Our computation for the shortest paths used Dijkstra's algorithm implemented with a quad-heap structure. An ellipse based strategy is utilized to limit the searching within a local ellipse or its MBR generated with the source and destination nodes. Detailed description of the strategy can be found in [6].

\subsection{Correlation Between Shortest Path and Euclidean Distance in Road Networks}

First, 1000 sample nodes were extracted systematically from each network to reconstitute sets $A$ and $B$, each containing 500 nodes. In the case of Alabama, all 952 nodes were used (Table 1). Every node in $A$ or $B$ would be regarded in turn as origins and destinations between which the shortest paths must be determined.

A set $R$ of ratios $r_{a b}=p_{a b} / e_{a b}\left(e_{a b}\right.$ : the Euclidean distance; $p_{a b}$ : the shortest path distance) could be computed for each sample set. In general, we could establish a real number $\tau$ as the threshold value for the elements in $R$ at a stated confidence level. A $\tau$ value at 95 percent would imply 95 percent confidence that the shortest path for a pair of nodes could be found within the extent built with the $\tau$ value. The $\tau$ values ranged from 1.238 (Texas) to 1.585 (Utah detailed) which appeared quite consistent across the networks.

\subsection{Numerical Premise}

We proposed the use of a minimum bounding rectangle (MBR) of ellipse to limit the search. Table 2 presented the average $r$-values against the 95 percent $\tau$ threshold of the ten networks. We would attribute a larger $\tau$ for California because of its elongated shape and Utah Detailed for its disrupted landscape. In other words, there is great likelihood (i.e. at least 95 percent of the time) that we could locate the shortest paths (SP) between any node pairs within the ellipse MBR. Our attempt to compare success-rates of the elliptical versus MBR search limits showed that the MBR is superior to the ellipse; the former had fewer SPs beyond bound. 
Table 2. $\tau$ values and comparison between elliptical and MBR limitation areas

\begin{tabular}{ccccccc}
\hline Network set & $\begin{array}{c}\text { Average } \\
\text { ratio } \\
r_{a b}=p_{a b} / e_{a b}\end{array}$ & $\begin{array}{c}\text { Threshold } \tau \\
\text { (at 95 \% } \\
\text { confidence) }\end{array}$ & $\begin{array}{c}\text { Number of } \\
\text { SP beyond } \\
\text { the ellipse }\end{array}$ & $\begin{array}{c}\text { Number of } \\
\text { SP beyond } \\
\text { the MBR }\end{array}$ & $\begin{array}{c}\text { Real } \\
\text { confidence } \\
\text { for ellipse } \\
\text { search }(\%)\end{array}$ & $\begin{array}{c}\text { Real } \\
\text { confidence } \\
\text { for MBR } \\
\text { search }(\%)\end{array}$ \\
\hline Alabama & 1.201 & 1.379 & 1588 & 840 & 99.30 & 99.63 \\
Georgia & 1.162 & 1.286 & 1013 & 619 & 99.59 & 99.75 \\
Pennsylvania & 1.179 & 1.393 & 761 & 453 & 99.70 & 99.82 \\
New York & 1.191 & 1.375 & 750 & 406 & 99.70 & 99.84 \\
Texas & 1.128 & 1.238 & 1220 & 369 & 99.51 & 99.85 \\
California & 1.170 & 1.426 & 1163 & 802 & 99.53 & 99.68 \\
Northeast USA & 1.166 & 1.334 & 438 & 250 & 99.82 & 99.90 \\
USA & 1.139 & 1.267 & 226 & 109 & 99.91 & 99.96 \\
Utah Detailed & 1.308 & 1.585 & 717 & 405 & 99.71 & 99.84 \\
Alabama & 1.209 & 1.334 & 822 & 549 & 99.67 & 99.78 \\
Detailed & 1.185 & 1.362 & - & - & 99.64 & 99.81 \\
Average & & & & & &
\end{tabular}

The last two columns in Table 2 recorded that more than $99 \%$ of the shortest paths could be identified within the limited areas. The higher confidence levels meant that the limited search was extremely effective because solutions to shortest paths could be found $99 \%$ of the time in one round of search. In other words, only a very small percentage of the shortest paths (an average of $0.36 \%$ for ellipse and $0.19 \%$ for MBR) would be found beyond the areas.

Table 3. Accuracy and efficiency of ellipse MBR limitation for solving shortest paths

\begin{tabular}{cccc}
\hline Network set & $\begin{array}{c}\text { Possibility of SPs } \\
\text { beyond the MBR but } \\
\text { near-SPs found (\%) }\end{array}$ & $\begin{array}{c}\text { Difference of near-SPs } \\
\text { found and real SPs (\%) }\end{array}$ & $\begin{array}{c}\text { Time saving with } \\
\text { ellipse MBR limitation } \\
(\%)\end{array}$ \\
\hline Alabama & 0.070 & 13.60 & 29.25 \\
Georgia & 0.108 & 6.94 & 34.41 \\
Pennsylvania & 0.069 & 9.10 & 16.44 \\
New York & 0.021 & 8.06 & 24.18 \\
Texas & 0.060 & 3.29 & 37.64 \\
California & 0.207 & 17.74 & 15.35 \\
Northeast USA & 0.077 & 7.46 & 32.83 \\
USA & 0.070 & 6.28 & 33.51 \\
Utah Detailed & 0.030 & 1.50 & 23.74 \\
Alabama Detailed & 0.026 & 2.56 & 35.73 \\
Average & 0.074 & 7.65 & 26.68 \\
\hline
\end{tabular}




\subsection{Establishing Accuracy and Computational Efficiency}

Our experiment showed that very few shortest paths (i.e. an average of $0.074 \%$ ) would be mis-calculated with the ellipse MBR limitation (i.e. an average of $7.65 \%$ longer than the actual shortest paths). The results indicated that the MBR method would not cause excessive loss in the computational accuracy.

Another experiment was conducted to verify the computational efficiency of the presented method for resolving the one-to-one shortest paths with the 1000 sample nodes (all 952 nodes for Alabama). It showed that an average $26.68 \%$ saving in computational time was realized with only minimal loss in accuracy (i.e. $0.074 \%$ of the optimum paths would be $7.65 \%$ longer than the actual shortest paths on average). The results were listed in Table 3.

\section{Conclusion}

The theoretically optimal solution and heuristic strategies could be integrated to offer a reasonable trade-off between efficiency and accuracy. The ellipse MBR method we put forth allows users to solve the shortest paths for a stated confidence and in less time. Our experiment showed that more than 99.8 percent of shortest paths could be found within the proposed MBR along with 26.68 percent time saving on average, with an average of only 0.074 percent of the optimal paths exceeding 7.65 percent of the lengths of the actual shortest paths.

\section{References}

1. Miller H.J. and Shaw S.L. Geographic Information Systems for Transportation: Principles and Applications. New York: Oxford University Press (2001).

2. Zhao Y.L. Vehicle Location and Navigation Systems. Boston: Artech House Publishers (1997).

3. Fisher P. F. A primer of geographic search using artificial intelligence, Computers and Geosciences, 16(1990) 753-776.

4. Car A. and Frank A. General principles of hierarchical spatial reasoning-the case of wayfinding, Proceedings of the 6th International Symposium on Spatial Data Handling, (1994) 646-664.

5. Holzer M., Schulz F., and Willhalm T., Combining speed-up techniques for shortest-path computations, Lecture Notes in Computer Science, 3059((2004) 269-284.

6. Feng Lu, Yanning Guan, An optimum vehicular path solution with multi-heuristics, Lecture Notes in Computer Science, 3039(2004) :964-971. 